\title{
Are antipsychotic drugs the right treatment for challenging behaviour in learning disability?: The place of a randomised trial
}

Richard Ashcroft, Bill Fraser, Michael Kerr and Zahir Ahmed Imperial College of Science, Technology and Medicine and University of Wales College of Medicine, Cardiff

\begin{abstract}
People with learning disability sometimes display challenging behaviour. This can be managed by use of antipsychotic medication or behavioural therapy or both. There is no solid evidence, however, that these therapies are safe and effective. A randomised controlled trial of antipsychotic medication has been proposed to deliver such evidence. However, this presents difficult issues in the ethics of research with learning disabled people. In particular, is a trial the most efficient and fairest way to evaluate practice in this area? This paper reviews the clinical situation, gives the rationale for the trial, and analyses the ethical arguments for and against such a trial.

(Fournal of Medical Ethics 2001;27:338-343)
\end{abstract}

Keywords: Learning disability; antipsychotic medication; randomised trial; challenging behaviour; research ethics

\section{The current clinical context}

LEARNING DISABILITY AND CHALLENGING BEHAVIOUR

Learning disability refers to individuals whose IQ is measured at less than 70 . Overall the prevalence of learning disability in the UK is between 1 and $2 \%$ of the population, ${ }^{1} 85 \%$ will have mild learning disability. Challenging behaviour is very common in this group, with about $20 \%$ of children and $15 \%$ of adults exhibiting it. ${ }^{23}$ The lower the IQ greater the prevalence of challenging behaviour, approaching $40 \%{ }^{4}$ in those with severe learning disability. Emerson $^{5}$ defined challenging behaviour as:

"Culturally abnormal behaviour(s) of such intensity, frequency or duration that the physical safety of the person or others is likely to be placed in serious jeopardy, or behaviour which is likely to seriously limit use of, or result in the person being denied access to ordinary community facilities".

Behaviour disturbance in individuals with learning disability stems from an interplay of two types of factor: environmentally independent factors, such as stage of development, preservation of homeostasis, habitual state of arousal of the individual, organic or functional mental illness, and environmentally dependent factors, such as aberrant behaviours that have been inadvertently reinforced, and aberrant, failed communication attempts. ${ }^{5}$ Behaviour disturbances can further be caused by major emotional universal needs in learning disabled people (for example security, friendship) being unmet.

The challenging behaviours exhibited include aggression, destructiveness, self injury and stereotypy. These can cause distress or harm to the individual, to their carers or to the general public.

CLINICAL MANAGEMENT

Managing individuals with challenging behaviour remains a very difficult task. A number of options are available. The most widely adopted is the use of psychotropic drugs, of which antipsychotic drugs are most frequently used. Other options are based upon behaviour modification techniques. These are usually carried out by specialised professionals, involve assessment of the behaviour, looking specifically at triggering factors and the impact of the behaviour on the environment, and then developing strategies to modify it. Antipsychotic drugs are by the far the easiest option to use in terms of cost, time and administration.

In learning disability there is a high use of antipsychotic drugs. About $22-45 \%$ of individuals residing in hospitals and $20 \%$ in the community will be taking antipsychotic drugs. ${ }^{6}$ The prevalence of psychiatric illness is between $8-15 \%{ }^{7}$ which means that $7-30 \%$ of all individuals taking antipsychotic drugs in institutions and $5-12 \%$ in the community setting, are doing so in order to manage their challenging behaviour alone. Between $9-26 \%$ of individuals taking antipsychotic drugs will be on more than one type of antipsychotic drug. The three commonest antipsychotic drugs used are thioridazine, chlorpromazine and haloperidol.

Not all individuals with challenging behaviour are prescribed antipsychotic drugs. When looking 
at factors determining drug use for the management of challenging behaviours, studies show that it is the effect of the challenging behaviour on the social environment which is likely to lead to use of drugs. ${ }^{8}$ In an institutional setting an individual with challenging behaviour has a greater chance of being prescribed an antipsychotic drug if he or she is violent and destructive, has a high level of physical skills, motor skills and self direction. ${ }^{9}$ Factors other than behaviour are also important in the use of antipsychotic drugs, including staff perception of the behaviour, environmental factors, staffing ratio and administrative treatment philosophies. ${ }^{10}$ Of the various challenging behaviours, aggression is the strongest predictor of use of antipsychotic drugs. ${ }^{11}$ Once started on antipsychotic drugs individuals are likely to remain on these for many years.

\section{SAFETY AND EFFECTIVENESS}

How useful antipsychotic drugs are in controlling challenging behaviour remains debatable. There are large numbers of publications addressing this issue; however, the vast majority of these are methodologically flawed or inconclusive. A recent review of the literature by Brylewski and Duggan ${ }^{12}$ as part of the Cochrane Database Systematic Review, showed over 500 citations assessing the impact of antipsychotic drugs on challenging behaviour. Of these only three were methodologically sound randomised controlled trials, but even these were unable to show whether antipsychotic drugs were beneficial or not in controlling challenging behaviour.

The usefulness of antipsychotic drugs in controlling challenging behaviour needs to be established because the use of these drugs is not without hazard. These drugs have both short term and long term side effects. Short term side effects are usually not a major problem as they are often self limiting, can be treated by using other drugs or dissipate on stopping the antipsychotic drugs. Idiosyncratic and long term side effects are of major concern. The idiosyncratic side effects are probably the most serious and associated with a significant mortality rate. The most common long term side effect is development of involuntary muscle movements, collectively termed tardive dyskinesia. This may lead to disfigurement and is often mistaken as characteristic of the underlying learning disability. Tardive dyskinesia will occur in $30-45 \%$ of individuals taking antipsychotic drugs on a long term basis. ${ }^{13}$ Tardive dyskinesia is a major problem because in $50 \%$ of cases it is irreversible even when the offending antipsychotic drug is discontinued.

Although not yet a problem in this country tardive dyskinesia has major litigation potential. In the USA there have been many successful litigations arising from the inappropriate use of antipsychotic drugs which has resulted in the development of tardive dyskinesia. The first of these, which was a milestone and responsible for opening the floodgate for other such cases, was Clites v State of Iowa
USA ${ }^{14}$ Clites was a 17 -year-old male with a learning disability who had been treated with thioridazine for sexual misconduct and aggression, by a consultant psychiatrist. The primary physician further increased the dose and added other antipsychotic drugs. There was no further contact from a psychiatrist for five years. No physical examination was performed for two and a half years. The antipsychotic drugs were continued despite deterioration in a fairly high functioning individual and the development of tardive dyskinesia. Clites was awarded $\$ 760,165$ as compensation. In view of potential litigation the use of antipsychotic drugs, which pose a serious risk to the individual, cannot continue without a defensible evidence base.

Another late onset side effect is tardive akathesia, with a prevalence between $7-18 \% .{ }^{15}$ This causes a subjective feeling of restlessness, which is often very uncomfortable and causes an individual to pace about and be irritable. Tardive akathesia can itself be mistaken for a challenging behaviour or may lead to an increased severity or frequency of an existing challenging behaviour.

Antipsychotic drugs have a big advantage in that they are very easy to use, provide a rapid calming and sedative effect, which can make an immediate impact on the challenging behaviour and compared to alternative strategies are very cheap and readily available. In many parts of the country there may be no professionals who are specifically trained to manage challenging behaviour using proven behavioural techniques, thus increasing the reliance on antipsychotic drugs.

RATIONALE FOR A CLINICAL TRIAL

Do antipsychotic drugs successfully control challenging behaviour in the short and long term?

A randomised controlled, double-blind, placebocontrolled trial would methodologically be the best way to proceed. Such a trial would need to be a multicentre trial in order to ensure that enough subjects are recruited for the trial to be able to provide a statistically significant result. Subjects would be randomised to receive either an antipsychotic drug or placebo. The current treatment philosophy is to use the minimum effect dose.$^{16}$ Subjects would be adults with learning disability who present with challenging behaviour. Subjects with a diagnosis of psychotic illness or well controlled challenging behaviour would be excluded from the trial, and all subjects would receive the normal behavioural and nursing care and social support. Changes in the subjects' behaviour, from the baseline, would be measured objectively using established techniques such as direct observation of their subject and environmental variables for a period of six months, with follow up at two years. ${ }^{\circ}$

\section{Ethical analysis of the proposed trial}

The following ethical issues arise in considering the proposed trial: benefit and risk to participants; resources, and consequences of not trialling. We do not here discuss consent issues, or the general problems involved in trials where patients are incapable of 
340 Research ethics: Are antipsychotic drugs the right treatment for challenging behaviour in learning disability?: The place of a randomised trial

giving consent. In the emergency setting of challenging behaviour, consent is normally impossible; where it can be obtained, it should be. It might be possible to obtain advance consent, although the status of such consent is not clear. These issues have been widely discussed elsewhere our concern here is to establish whether the tria would be in the best interests of the participants, which is the determinant issue for enrolling the majority of the proposed subjects at the time of their challenging behaviour. ${ }^{17} 18$

\section{BENEFIT TO PARTICIPANTS}

This should be considered in two stages: the benefit to participants of a trial, and the simple benefit of receiving antipsychotic medication as a treatment for challenging behaviour. The issues around the trial are simpler, and are discussed first.

\section{Ethical aspects of the trial}

The classic clinical trial has two features: first that a new treatment is compared with a standard treatment (which in some cases is a placebo), and second the risks are supposed to be "proportionate" (ie, reasonable) to the benefits. The proposed trial differs from the classic trial in both respects.

Firstly, there is no "new" treatment; the "standard" treatment is novel only from an evidencebased medicine viewpoint, according to which "new" is synonymous with "unevaluated". The question arises of whether the participant could be worse off by participating in the trial than he or she would otherwise be. At present, those patients whose challenging behaviour is treated with antipsychotics are treated that way because the intended benefits to them of controlling their behaviour outweigh the harms of the side effects. Such treatment is currently common, but not universal, as our review of management shows. In a placebocontrolled trial, if antipsychotic therapy "works", then the participants receiving the placebo would be worse off than they would be usually. If it "doesn't work", then those receiving the antipsychotics would be no worse off than usual, and those receiving the placebo would be better off than usual (at least as regards side effects). Participants in the proposed trial can be included if they are not currently using antipsychotics, so that they are not missing out on treatment which has been found to be effective for them. As in the debate on trials in schizophrenia, difficulties arise when patients who are taking medication which is effective for them are asked to undergo randomisation or a "washout" period which may lose them benefits of symptom control. This issue should not arise here, as with each new patient we do not know which treatment is actually more effective for them.

The second question concerns proportionality. We just assumed that the side effects were reasonable, given the severity of challenging behaviour and the benefits of controlling it. But it is clear that this cannot be merely assumed. Assume now that the intended benefit (control of challenging behaviour) is worthwhile for the participants. How can we trade off benefit against harm? Normally we assume that patients do this for themselves when they consent to (or refuse) the treatment. In this case we may not be able to rely on consent, for two reasons, first the capacity of the patient to consent may be diminished (both by the complexity of the trial and by their learning disability) and second because the treatment is initiated in a crisis situation against the will of the patient. It may be possible to obtain consent in advance of episodes of challenging behaviour and treatment, but this could be difficult. So we must decide whether, all things considered, this is in the best interests of the patient. ${ }^{18-20}$ Since we do not know whether the intended benefit will be actual benefit, we must concentrate on the intended benefit.

A third issue is whether patients enrolled in this trial can be withdrawn from the trial if their welfare is adversely affected by the treatment they receive in the trial. Patients within the trial will be subject to rather closer monitoring of their health than normal, and should have rapid access to behavioural and other treatments according to need, aside from the placebo/antipsychotic treatment they are assigned in the trial. Criteria must be established for (a) what rescue treatments are available to patients, (b) when they can or should be withdrawn from the trial, (c) what stopping rules are to be applied in case patients in one arm of the trial appear to be doing markedly better or worse than the other.

\section{Ethical aspects of the treatment}

Now assume that antipsychotic medication is effective in attaining the primary endpoint, control of challenging behaviour. We are still left with two questions: how does the treatment achieve this effect, and whether it is morally right to achieve this effect and to do so in this way.

How the treatment achieves its effect is not clear, although the continued use of the treatment suggests there is some relevant effect. Antipsychotic drugs are known to have pharmacological action as dopamine receptor antagonists and possibly also as seratonin receptor antagonists. But little is known about how this pharmacological action controls challenging behaviour.

Challenging behaviour is clearly problematic for the carer and for the patient in some sense, and normally we would agree that solving problems of this kind is a good thing, and beneficial to the patient. Given the danger and distress caused to the patient and his or her carers by challenging behaviour, and the difficulties in managing it, it might be reasonable to use drugs simply because they do seem to control the behaviour. Worrying about how they do so might seem to be a philosophical luxury. If we have reason to suppose, however, that the challenging behaviour is caused by external stressors (from boredom to unhappiness, and possibly involving neglect or maltreatment), then there are serious and difficult questions to answer about the use of drug therapies. For example, it could be 
argued that we are merely suppressing the challenging behaviour, rather than addressing its causes. If this were true, then a consistent stress, leading to consistent challenging behaviour, would lead to long term maintenance on antipsychotic drugs, rather than to removal or palliation of the cause of stress. The first line of justification must be the best interests of the patients themselves, normally understood to mean the "medical best interests". There seems to be doubt over what this means for patients in these circumstances, and over whether this treatment promotes these interests.

A second justification might be involved, which is that use of the drug is intended to prevent the patient causing harm to him or herself or others in the short term. This is a commonly agreed justification for chemical or physical restraint, but it derives from the emergency nature of the circumstances. ${ }^{21}$ Only if no other means can be found to reduce the long term risk of dangerous challenging behaviour, and thus as a last resort, does this become a justification for long term or routine use, and careful guidelines would be required for use. Learning disabled people are not generally incapable of learning how to express emotion and behave appropriately (although a few very severely disabled people may be). In order to learn how to do this, some challenging behaviour would be expected; immediate suppression of this behaviour would make learning impossible. In some cases, resource constraints may make this attitude difficult for carers to apply (see below). This would be an argument for more resources, on justice grounds, rather than making a policy of drug treatment for non-psychotic patients. Fostering learning would promote the longer term goal of a good life for the learning disabled person as a moral agent, able to take (some) responsibility for their own behaviour and wellbeing.

A third, indirect justification for use would be that an indirect danger to patients is that their challenging behaviour lowers their chances of independent living and risks their relationship with their carer. Hence, the patient benefits from undergoing this treatment because of the social impact of removing the challenging behaviour. This is identical in form to the trade off that schizophrenic and bipolar disorder patients face with their medication-taking the side effects of medication as the price to pay for an orderly and stable social life. As we know, many such patients do not choose to pay this price when they have a choice (although many do). Treatment of emotional distress or social problems by psychopharmacologic means is hugely controversial.

A fourth, indirect justification for use would be that learning disability is not well understood by the public at large, challenging behaviour in the community is not normally tolerated, and as a group people with learning disability suffer from stigma and prejudice if the association between learning disability and challenging behaviour is thought by the ill-informed to be both common in this group and offensive. While it would be preferable to improve social understanding and tolerance, in the context of community care generally, this may be a utopian dream. Hence, our only practical option may be to control the challenging behaviour as best we can, rather than to try to change social attitudes toward it and toward learning disabled people. So even if antipsychotic treatment is harmful to the individual, it may be comparatively less harmful to the individual than the effects of stigma and intolerance attendant upon uncontrolled behaviour. This is a sensible view, although it is controversial, and not all interested parties agree on this response. A comparable debate exists on the appropriateness of cosmetic surgery to remove the characteristic facial features of children with Down's, to "protect" them from the stigmatising attitudes of others. The analogy is not, however, exact, since in the case of challenging behaviour the individual may suffer direct harms from himself or his carers in addition to more indirect social harms.

It is therefore not entirely clear whether the intended benefit to the patient is actually a benefit, and actually to the patient, rather than to the carer or others. The harms of side effects to the patient of the treatment are clear. However, we should not underestimate the harm risked to the patient, his or her carers and others by leaving such challenging behaviour untreated or unmanaged. This harm is widely regarded as significant, although difficult to quantify. The question is whether the antipsychotic treatment is a reasonable approach to these problems, and only thereafter does the question of whether it is effective arise. Does antipsychotic medication deal with the cause of the challenging behaviour, or only its expression? Dealing with the expression only could be acceptable if removing the cause was (a) impossible or (b) impractical under the circumstances (crisis management) or (c) an expensive or unreasonable use of resources which would damage the care of others. Of these, (a) is an open question under the proposed protocol, and may be impossible to answer in the near future; (b) is only a satisfactory answer if there were parallel, effective means for reducing or controlling the likelihood of such "crises". The principal rationale for the trial therefore seems pragmatic, depending on the difficulty of changing the carer's or the purchaser's behaviour in the absence of randomised controlled trial (RCT) evidence for ineffectiveness of antipsychotics, or on resource questions.

\section{RESOURCES}

We might argue that other treatments or management techniques which seek to address the psychological cause of challenging behaviour are betterfor example behavioural techniques. This is not, however, the only consideration. First, we have no evidence that these treatments are any more effective regarding the primary endpoint of reducing or controlling challenging behaviour. So we cannot switch from one unproven treatment to another. Between two unproven treatments, and in the absence of evidence or the prospect of getting any, 
342 Research ethics: Are antipsychotic drugs the right treatment for challenging behaviour in learning disability?: The place of a randomised trial

it may be that choosing on "philosophical" grounds is quite as reasonable as use of any other grounds. That is, if the "philosophy" of the particular care unit is to concentrate on behavioural change rather than psychopharmacology, that may be reasonable in the absence of other compelling arguments-but so would the opposite preference. Moreover, a treatment which explicitly aims at promoting a patient-centred good (emotional wellbeing) rather than crisis management or society- or carer-centred goods (stable behaviour) would normally be regarded as morally preferable. However, this would be glib. Have we grounds for supposing that behavioural techniques are effective in promoting emotional wellbeing? Have we reason to suppose that these methods, which are staff-intensive and demanding, are a reasonable use of resources in the context of current mental health care practice? We might argue that while non-antipsychotic methods are preferable from the point of view of autonomy, they could not be justified from the point of view of just use of resources. For example, the cost of providing emotional support might be such that there remain no resources for other activities which contribute to the patient's wellbeing-for example, occupational therapy. Alternatively, other patients' care and support might be diminished in order to resource behavioural therapy for challenging behaviour.

We can prefer one treatment to another on various grounds: treatment $\mathrm{A}$ is better than treatment $\mathrm{B}$ because it is (a) more effective, (b) cheaper or (c) less dangerous or risky. There are various ways of combining these three criteria. However, we would not normally prefer treatment A to treatment B if A was of unproven benefit, expensive and dangerous unless treatment B was prima facie even worse. One difficulty here is that price and risk are relative concepts. This makes clear arguments about the justice of purchasing behavioural rather than antipsychotic treatments difficult to ground. (Note that in many circumstances antipsychotic treatments are used in addition to behavioural ones, but as the distribution of personnel trained in behavioural methods is sparse, so we can assume that the choice is between behavioural and psychopharmocological treatments).

CONSEQUENCES OF NOT TRIALLING

Our review of clinical management identifies a widespread practice which is of dubious safety to patients and which lacks compelling evidence for (or against) its efficacy. Some might regard this as sufficient demonstration that the use of antipsychotics in the non-psychotic learning disabled person is unjustified and should be stopped. Is a randomised controlled trial the only or best way to change practice?

Note first of all that a clinician who regards a treatment as not beneficial to his or her patient is obliged not to use it. But the situation may be more vague: the clinician may think other treatments-not available to him or her for one reason or another-are better, but that this treatment, which is available, is still better than nothing. Alternatively, it may be that recognising that this treatment is not ideal theoretically, nothing better is actually available at present. This would appear to be the case here. This would be a local, temporary justification for use. As a matter of policy, there is some sort of obligation to evaluate whether the other treatments should be made available and to evaluate whether the current standard is actually better or worse than nothing. This argument may be strengthened by appeal to the diversity of opinion in the professions (clinical equipoise) and to the lack of current research evidence for this population.

If the arguments about the moral status of using this kind of treatment have weight independently of the evidential arguments, then it may be that use of antipsychotics for non-psychotic people with learning disabilities is morally wrong independently of their "effectiveness". However, analogous to clinical equipoise, it is arguable that there is moral difference of opinion in this context, and consequently the only reliable way to support or to change the current practice will be to complete a clinical trial.

If this is plausible, there is a further moral difficulty. It is arguable that we are using a group of patients to settle a difference of scientific opinion, and to help future patients (including these patients themselves in the future), rather than to aid these patients now. If it is morally wrong to treat these patients in this way, then we should just stop doing so. This argument reduces to the statement at the start of the previous paragraph: a clinician must use his or her clinical judgment. Arguably, this argument is not symmetrical: a clinician who believes that it is morally right to use this treatment must meet a higher standard of proof, because he or she must further show that the treatment works. The clinician who morally objects arguably does not need to show further that the treatment does not work. However, if the basis of his or her objection is in effect that the treatment is ineffective then he or she does have an obligation to show why he or she believes this, based on clinical evidence.

Thus, there are moral arguments for changing practice as well as evidential ones. As a matter of health policy, however, it is arguable that these should be grounded on common evidential standards, since these are public and subject to scientific scrutiny. However, this argument is very difficult to make secure. It seems to be "utilitarian" in the sense of seeking to maximise total welfare, disregarding the question of whether the trial participants are made better or worse off individually. Competent adult patients are capable of choosing to benefit others at the cost of some risk (possibility of harm) to themselves; adults with learning disability are arguably not able to do so, and it is very difficult to say whether medical professionals would be able to show that for any given individual it was in their best interests to participate in this trial. ${ }^{22}{ }^{23}$

Notwithstanding the above arguments, if the patients enrolled in the trial are currently not being treated, or being treated unsuccessfully, it is 
arguable that for these patients randomisation within a placebo-controlled trial is not contrary to their interests. In other words, there is no tension for these individuals between treatment in their best interests, and obtaining scientific evidence about the effectiveness of the treatment under trial. ${ }^{24}$

\section{Conclusion}

The grounds for the trial proposed are, on balance, ethically sound. The more complex issues of resources and of the medical nature of challenging behaviour and the basis for therapeutics are very important, and should guide health policy and medical research in this area, but are somewhat imponderable in the short term, and this trial presents one way of ensuring that the patients' best interests are served.

It would be essential for the trial to proceed with the understanding and cooperation of the parents, relatives and/or carers of the patients, and with the involvement of the patient support groups.

In conclusion: is a trial of antipsychotic treatment for challenging behaviour in learning disability morally acceptable? Yes-provided the very idea of drug treatment for behavioural problems is.

\section{Funding}

This paper was prepared on the basis of work commissioned by the UK NHS Health Technology Assessment Programme (pharmaceutical panel).

Richard Ashcroft is Sir Siegmund Warburg Lecturer in Medical Ethics, Medical Ethics Unit, Department of Primary Health Care and General Practice, Imperial College of Science, Technology and Medicine, London. Bill Fraser is Professor of Psychiatry of Learning Disability, Welsh Centre for Learning Disabilities Clinical Studies, University of Wales College of Medicine, Cardiff. Michael Kerr is Senior Lecturer and Honorary Consultant Psychiatrist at the Welsh Centre for Learning Disabilities Clinical Studies. Dr Zahir Ahmed is Specialist Registrar and Honorary Lecturer in Learning Disability at the Welsh Centre for Learning Disabilities Clinical Studies.

\section{References}

1 Bregman JS, Harris JC. Mental retardation. In: Kaplan HI, Sadock BJ, eds. Comprehensive textbook of psychiatry [6th ed]. Baltimore: Williams \& Wilkins, 1995: 2207-41.

2 Sigafoos J, Elkins J, Kerr M, Attwood T. A survey of aggressive behaviour among a population of persons with intellectual disability in Queensland. fournal of Intellectual Disability Research 1993;38:369-81.
3 Harris P. The nature and extent of aggressive behaviour amongst people with learning difficulties (mental handicap) in a single health district. Fournal of Intellectual Disability Research 1993;37:221-42.

4 Collacott RA, Cooper SA, Branford D, McGrother C. Epidemiology of self-injurious behaviour in adults with learning disabilities. British fournal of Psychiatry1998;173:42832 .

5 Emerson E, Bromley J. The form and function of challenging behaviours. Fournal of Intellectual Disability Research 1995;39: 388-98.

6 Ahmed Z, Fraser W, Kerr M, Kiernan C, Emerson E, Robertson J, et al. The effects of reducing antipsychotic medication in people with a learning disability. British fournal of Psychiatry people with a lear

7 Deb S, Fraser WI. The use of psychotropic medication in people with learning disability: towards rational prescribing. Human Psychopharmacology, 1994;9:259-72

8 Kiernan C, Reeves D, Alborz A. The use of anti-psychotic drugs with adults with learning disabilities and challenging behaviour. Fournal of Intellectual Disability Research 1995;39: 263-74.

9 Intagliata J, Rinck C. Psychoactive drug use in public and community residential facilities for mentally retarded persons. Psychopharmacology Bulletin 1985;21:268-78.

10 Harper DC, Wandsworth JS. Behavioural problems and medication utilisation. Mental Retardation 1993;31:97-103.

11 Fraser WI, Leudar I, Gray J, Campbell I. Psychiatric behaviour disorder in mental handicap. Fournal of Mental Deficiency Research 1986;30:49-59.

12 Brylewski J, Duggan L. Antipsychotic medication for challenging behaviour in people with learning disability. Fournal of Intellectual Disability Research 1999;43:360-71 (and Cochrane Datalectual Disability Research 1999;43:360-71 (

13 Kalachnik JE, Harder SR, Kidd-Nielsen P, Errickson E, Doebber M, Sprague R. Persistent tardive dyskinesia in randomly ber $M$, Sprague $R$. Persistent tardive dyskinesia in randomly non-neuroleptic history groups. Psychopharmacology Bulletin 1984;20:27-32

14 Rinck C, Guidry J, Calkins CF. Review of states' practices on the use of psychotropic medication. American fournal of Mental Retardation 1989;93:57-668.

15 Ganesh S. Murti Rao J, Cowie VA. Akathesia in neuroleptic medicated mentally handicapped patients. Fournal of Mental Deficiency Research 1989;33:323-9.

16 Duggan L, Brylewski J. Effectiveness of antipsychotic medication in people with intellectual disability and schizophrenia: a systematic review. Fournal of Intellectual Disability Research 1999:43:94-104 (and Cochrane Database Systematic Review 2000;2: CD000030).

17 National Bioethics Advisory Commission. Research involving subjects with mental disorders that may affect decision-making capacity. Rockville, MD: National Bioethics Advisory Commission, 1998 .

18 Law Commission Report 231. Mental incapacity. London: Law Commission of England and Wales, 1995.

19 Medical Research Council. The ethical conduct of research on the mentally incapacitated. London: Medical Research Council, 1991.

20 F v W Berkshire Health Authority, [1989] 2 All ER 545.

21 Montgomery J. Health care law. Oxford: Oxford University Press, 1997: ch 13.

22 Edwards SD. An argument against research on people with learning disabilities. Medicine, Health Care and Philosophy 2000; 3:69-73.

23 Freedman RI. Ethical challenges in the conduct of research involving persons with mental retardation. Mental Retardation 2001;39:130-41.

24 Ashcroft RE. Giving medicine a fair trial. British Medical fournal 2000;320:1686. 